\title{
Behaviour of Paramecium aurelia in Solutions of Purified and Fluorescent Labelled Tetanus Neurotoxin
}

\author{
By S. I. ZACKS AND M. F. SHEFF \\ Ayer Clinical Laboratory and Department of Neurology, \\ Pennsylvania Hospital, Philadelphia, Pennsylvania, U.S.A.
}

(Received 23 November 1965)

\begin{abstract}
SUMMARY
An investigation of the effects on Paramecium aurelia of crude and a highly purified tetanus neurotoxin preparation free from oxidase and haemolysin activity was made to assess the value of this organism in a bioassay system and as a convenient organism for the study of the mode of action of the toxin. Whereas crude tetanus toxin produced cytotoxic changes in $\boldsymbol{P}$. aurelia, purified tetanus neurotoxin did not even when the paramecia were exposed to ten human lethal doses.

Incubation of the paramecia with fluorescent-labelled toxin revealed that it accumulated within food vacuoles and in focal areas of cytoplasm but not in the cell membrane. Cytologic studies of the paramecia by means of light and electron microscopy revealed no abnormalities.
\end{abstract}

\section{INTRODUCTION}

Recent studies by Roux \& Serre $(1962,1964 a, b)$ have demonstrated cytotoxic effects on Paramecium aurelia exposed to partially purified tetanus toxin. If paramecia were specifically sensitive to tetanus neurotoxin in low concentrations, they would be valuable for bioassay of toxin fractions and serve as convenient material for the study of the mode of action of the toxin using cyto- and biochemical methods. For these reasons, the action of crude and highly purified tetanus toxin on $\boldsymbol{P}$. aurelia was investigated.

\section{METHODS}

Paramecium aurelia containing cytoplasmic DNA was cultured by the method of Sonneborn (1947) and fed with Aerobacter aerogense. In the majority of the experiments, actively swimming organisms were used 12-24 $\mathrm{hr}$ after the last feeding.

Toxin preparations. Three preparations of tetanus toxin were utilized in these experiments.

1. Crude toxin; this was a filtrate of Clostridium tetani cultures obtained from Wyeth Laboratories, Radnor, Pennsylvania, containing 600,000 m.l.d./ml. In our assay system, one mouse m.l.d. was equal to $2.5 \times 10^{-6} \mathrm{~m} . \mathrm{s}$.d. (minimum saturation dose: Sheff, Perry \& Zacks, 1965). This material (referred to as crude toxin) contained 12-16 protein components, only one of which has neurotoxic activity (Sheff et al. 1965). The crude toxin also contained a highly active oxidase and was capable of partly uncoupling oxidative phosphorylation in isolated liver mitochondria (Sheff \& Zacks, unpublished). 
2. Purified toxin; this purified neurotoxin was obtained by chromatography on DEAE cellulose according to the method of Sheff et al. (1965) and had a m.s.d. of less than $10 \mu \mathrm{g}$./mouse, a dose equivalent to $4 \times 10^{4} \mathrm{~m} . \mathrm{l}$.d. This material was free from oxidase and haemolysin activity. Stock solutions containing $10 \mu \mathrm{g}$. $/ \mathrm{ml}$. were made up in $\mathrm{NaCl}$ solution $(0 \cdot 85 \mathrm{~g} . / 100 \mathrm{ml}$.) and serially diluted before addition to the paramecia.

3. Fluorescent labelled toxin; purified tetanus toxin was labelled with rhodamine B200 chloride by the method of Rinderknecht (1962) without significant loss of activity. All unreated rhodamine was removed by column chromatography on G-25 Sephadex.

In some experiments, toxin was added directly to $10 \mathrm{ml}$. samples of the culture containing both bacteria and protozoa, but in most of the experiments the bacteria were removed before adding the toxin by sedimentation of the paramecia at $1000 \mathrm{~g}$. The protozoa were then sucked up with a Pasteur pipette and placed in culture medium free from bacteria. To observe the action of the tetanus toxin on the protozoa, approximately 50 actively swimming Paramecia aurelia were placed in small partially-covered watch glasses containing $10 \mathrm{ml}$. culture water. After a $60 \mathrm{~min}$. period of temperature equilibration at $25^{\circ}, 0.1 \mathrm{ml}$. of toxin preparation in saline was added by means of a syringe. Doses of 0.12-8 m.s.d. (0.0012-0.080 mg. toxin/ml.) were tested. After addition of the toxin, the paramecia were observed at $5 \mathrm{~min}$. intervals for the first few hours and then intermittently up to $24 \mathrm{hr}$. The

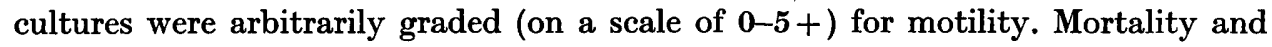
morphological abnormalities were looked for under stereomicroscopic magnifications of $\times 10-30$. The microscopic illumination was turned off between observations to avoid undue heating of the paramecia. The temperature remained at the range of $\mathbf{2 4}^{\circ}$ to $28^{\circ}$ throughout most of the experiments; in a few experiments, the temperature was maintained at $37^{\circ}$.

Cytologic methods. After the bacteria-free cultures of paramecia were exposed to the action of tetanus toxin and observed for several hours, the protozoa were concentrated by slow speed centrifugation to a volume of $0.5 \mathrm{ml}$. and fixed by the addition of $40 \%$ formaldehyde to obtain a final concentration of $10 \%(\mathrm{v} / \mathrm{v})$ or with $25 \%$ glutaraldehyde to obtain a final concentration of $6 \%(v / v)$ for fine structure studies. The fixed paramecia were washed and embedded in paraffin or Epon 812 by standard methods.

Fluorescent-labelled tetanus toxin. After exposing the protozoa to rhodaminelabelled toxin for various times, the paramecia were collected with a Pasteur pipette and examined in a Reichert ultraviolet microscope using a cardioid darkfield condenser. An ultraviolet pass filter IS/UGL was used at the ultraviolet source and 2XI/GG and Wratten 2B filters were used at the eyepiece. In some experiments, after exposure to labelled toxin, the paramecia were killed by osmium tetroxide or glutaraldehyde vapour and examined in ultraviolet radiation.

Detoxification. As it was possible that the paramecia were capable of detoxifying the tetanus toxin; the incubation medium was tested for toxicity after the paramecia had been exposed to the toxin for 6-24 $\mathrm{hr}$. The protozoa were sedimented a $1000 \mathrm{~g}$ and samples of the supernatant fluids were injected into mice which were then observed for signs of tetanus intoxication. 


\section{RESULTS}

\section{Effects of crude tetanus toxin}

One to two minutes after addition of crude tetanus toxin to actively swimming paramecia, we observed a consistent slowing of their swimming. The cilia continued to beat and they continued to swim in a characteristic spiral pattern but at decreased velocity. Parallel control preparations showed free and active swimming. The organisms in the toxin solution also tended to hug the periphery of the watch glass and many organisms were immobile by 3-4 min. after addition of the toxin. By 30-40 min. all the organisms were dead and many showed clear blebs projecting from the periphery of the organisms similar to those described by Roux \& Serre (1962). The control protozoa continued to swim at their initial velocity.

\section{Effects of purified unlabelled toxin}

When highly purified tetanus toxin in a concentration from $\mathbf{0 . 2 5}$ to $8 \mathrm{~m} . \mathrm{s} . \mathrm{d} . / \mathrm{ml}$. was added to freely swimming paramecia, there was an initial slowing of swimming beginning 5-20 min. after exposure of the organisms to the toxin; this was followed by partial recovery. With the highest concentration $(8 \mathrm{~m} . \mathrm{s.d}$.$) , onset of decreased$ activity occurred after $5 \mathrm{~min}$; ; and after partial recovery at $20 \mathrm{~min}$., there was a second period of slowing followed by partial recovery (Fig. 1 A). Complete recovery had not occurred at the time the experiment was terminated. Paramecia in less concentrated toxin solutions $(0 \cdot 25-1 \mathrm{~m} . \mathrm{s} . d$.$) required 15 \mathrm{~min}$. to show decreased swimming activity and demonstrated a tendency to recover after $5 \frac{1}{2} \mathrm{hr}$ of exposure to the toxin solutions (Fig. 1 B, C). Paraffin ( $7 \mu$ ) and Epon $(1 \mu)$ embedded sections from the sedimented paramecia collected at the termination of this experiment did not show any cytological abnormalities when examined either by light or electron microscopy.

When paramecia were kept for $24 \mathrm{hr}$ in purified toxin solutions containing 0.5-0.25 m.s.d., occasional dead organisms were seen but not cytotoxic changes such as swelling or vacuolization were observed. In one experiment, ten human lethal doses $(\mathbf{0} \cdot \mathbf{1} \mathbf{m} . \mathbf{s} . d$.) was added to the paramecia and the preparation was observed for $24 \mathrm{hr}$. At the end of this period, they continued to swim actively without evidence of cytotoxic changes.

When samples of culture medium obtained after incubating paramecia with high concentrations of tetanus toxin (0.1-0.5 m.s.d.) was injected into groups of mice, all the mice died with signs of generalized tetanus in the time expected for equivalent doses of fresh purified neurotoxin.

\section{Effects of fuorescent-labelled tetanus toxin}

When doses of rhodamine-labelled purified tetanus toxin (0.1-1.0 m.s.d.) were added, the protozoa showed an initial slowing reaction as before but there was no swelling, vacuolization or death of organisms. When examined under ultraviolet radiation in the fresh state or after fixation with glutaraldehyde vapour, one to three vacuoles (10-12 $\mu$ diam.) filled with fluorescent label were observed (Pl. 1, fig. 1). These vacuoles corresponded in appearance and location with the food vacuoles observed in sectioned organisms. The cell membrane showed no labelled toxin but focal areas of the cytoplasm contained fluorescent-labelled toxin adjacent to the 
food vacuoles. Paramecia which had not been exposed to fluorescent toxin showed diffuse blue autofluorescence of the cytoplasm and bright blue fluorescence in the macronucleus. Bright yellow autofluorescent granules (1-2 $\mu$ ) were present in the cytoplasm and, in some areas, formed aggregates measuring up to $7 \mu$ ( $\mathrm{Pl}$. 1, fig. 2).
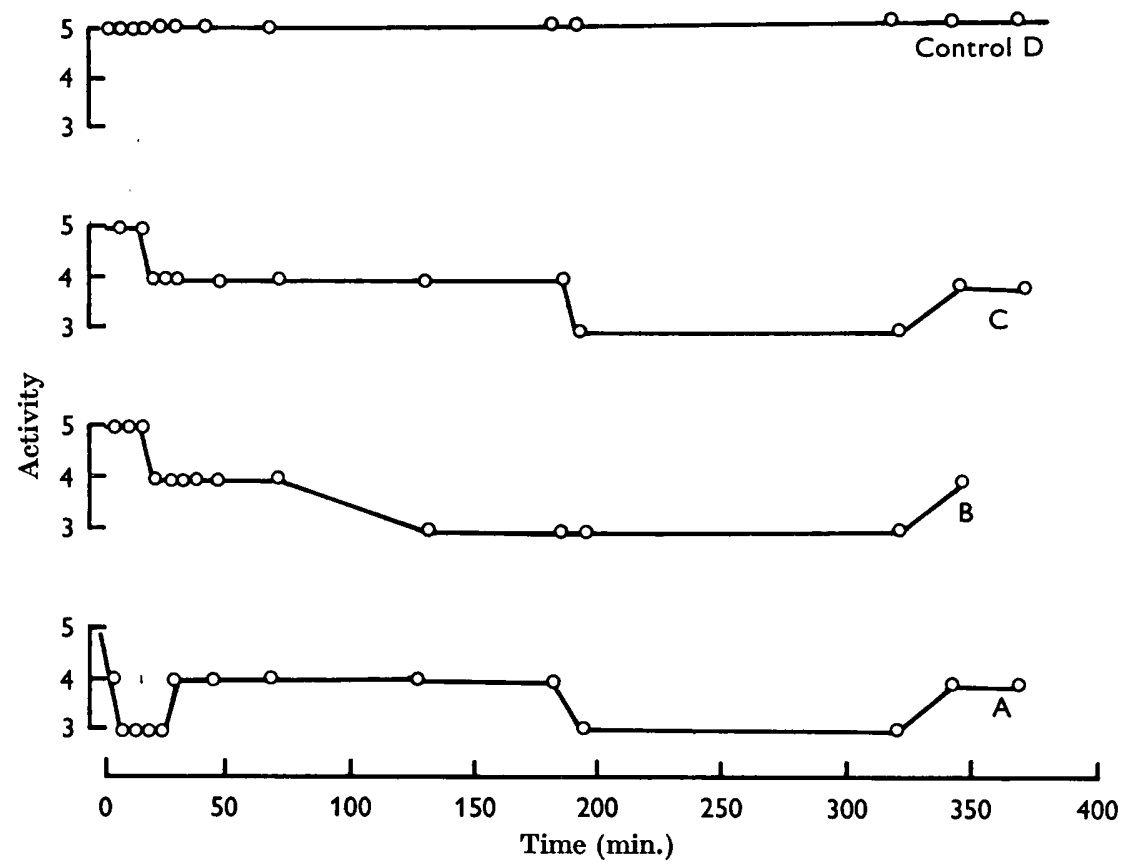

Fig. 1. A. Graph showing the effect of purified tetanus neurotoxin (8 m.s.d.) on the motility of Paramecium aurelia. Note the prompt decrease in swimming velocity followed by partial recovery.

B. Graph showing the effect of a lesser concentration of tetanus neurotoxin (1 m.s.d.) on the motility of Paramecia.

C. Graph showing the effect of tetanus neurotoxin $(0.25$ m.s.d.) on the motility of paramecia. Note the delayed onset of slowing of swimming and the tendency to recover.

D. Graph showing the motility of the control group of paramecia observed at the same time as the paramecia exposed to tetanus toxin (A, B, C). Activity is expressed on an arbitrary scale of 5 to 0 , with 5 representing the motility of non-intoxicated paramecia.

\section{DISCUSSION}

This work has clearly shown that although crude tetanus toxin preparations containing numerous components had a cytotoxic effect on paramecia, a highly purified preparation which contained the neurotoxic principle of tetanus toxin does not. When exposed to crude toxic preparations paramecia showed swelling, bleb formation and cell death as reported by Roux \& Serre (1962). We have previously observed marked swelling of isolated liver mitochrondria in vitro when they were exposed to similar concentrations of crude toxin (Zacks \& Sheff, 1963). However, purified tetanus neurotoxin failed to produce mitochondrial swelling.

Cytologic changes were not found in the poisoned paramecia. The cytoplasmic mitochondria did not show abnormalities that have previously been observed in mouse and human muscle in tetanus poisoning (Zacks \& Sheff, 1964; Zacks, Hall \& Sheff, 1966). 
Since the purified tetanus toxin solutions retained their lethality when injected into mice after incubation with paramecia, it is clear that the lack of cytotoxic changes during exposure to high concentrations of purified toxin was not due to destruction of toxin by the protozoa.

Experiments with fluorescent-labelled purified toxin demonstrated accumulation of the toxin within vesicles interpreted as food vacuoles and in focal areas of the cytoplasm. There was no binding to the pellicle. Beale \& Kacser (1957), using fluorescent-labelled antibodies to cilia proteins, found fluorescent labelled protein within food vacuoles and on the cell surface.

It would seem that the results of Roux \& Serre $(1962,1964 a, b)$ were probably due to a component of the crude tetanus toxic preparation used, rather than to the active neurotoxic principle itself. It is not surprising that Roux \& Serre (1962) were able to show that tetanus antitoxin prevented the effects shown by their toxin preparations, because the usual commercial tetanus antitoxin preparation contains a large number of antibodies against many components of crude toxin as well as antibodies against the neurotoxic component (Sheff et al. 1965). We conclude that Paramecium aurelia cannot be used as a sensitive bioassay for tetanus neurotoxin.

I wish to thank Professor John R. Preer, Jr., of The University of Pennsylvania for supplying the initial samples of paramecia and information on their culture. Also, we wish to acknowledge the technical assistance of Miss Marilyn Salscheider and Mrs Steven Weiss and Miss Vicki Lavalle who helped in the preparation of the manuscript. This investigation was supported by a grant to The Pennsylvania Hospital by the John A. Hartford Foundation, Inc., New York.

\section{REFERENCES}

Beale, G. H. \& Kacser, H. (1957). Studies on the antigens of Paramecium aurelia with the aid of fluorescent antibodies. J. gen. Microbiol. 17, 68.

RiNDERKNECHT, H. (1962). Ultra rapid fluorescent labelling of proteins. Nature, Lond. 193, 167.

Roux, J. \& Serre, A. (1962). Action de la toxine tétanique sur la cellule de protozaire. Etudes sur Paramecium aurelia. Ann. Inst. Pasteur, 103, 623.

Roux, J. \& Serre, A. (1964a). Action de la toxine tetanique sur Paramecium aurelia. Facteurs influencant l'action de la toxine et réversibilitie de la fixation. Ann. Inst. Pasteur, 107, 635.

Roux, J. \& Serre, A. (1964b). Obtention de lignes de Paramecies sensibilises a la toxine tetanique. Ann. Inst. Pasteur, 107, 647.

Sheff, M. F., Perry, M. B. \& ZACks, S. I. (1965). Studies on tetanus toxin II. The preparation of purified tetanus toxin and its fluorescent labelling. Biochim. biophys. Acta, 100, 215.

Sonneborn, T. M. (1947). Recent advances in the genetics of Paramecium and Euplotes. Advanc. Genet. 1, 264.

Zacks, S. I. \& SheFF, N. F. (1964). Studies on tetanus toxin I. Formation of intramitochondrial dense granules in mice acutely poisoned with tetanus toxin. $J$. Neuropath. exp. Neurol. 23, 306.

ZACKs, S. I. \& ShEFF, M. F. (1965). Studies on tetanus toxin III. Intercellular localization of fluorescent-labelled tetanus toxin and antitoxin in mice. Acta Neuropath. 4, 267.

ZACKs, S. I., HALl, J. A. S. \& ShefF, M. F. (1966). Studies on tetanus IV. Intramatichondrial dense granules in skeletal muscle from human cases of chronic tetanus intoxication. Am. J. Path. (in the Press). 


\section{EXPLANATION OF PLATE}

Fig. 1. Photomicrograph of a paramecium prepared as a whole mount and photographed in ultraviolet light. The bright objects (arrow) are yellow autofluorescent lipochrome granules. The cytoplasm shows pale autofluorescence. The line indicates $16 \mu$.

Fig. 2. Photomicrograph of a paramecium prepared as a whole mount and photographed in ultraviolet light after incubation in purified tetanus neurotoxin labelled with Rhodamine. The three bright objects (arrows) represent concentrations of red fluorescent tracer and the smaller bright objects in the cytoplasm are yellow pigment granules. The line indicates $15 \mu$. 


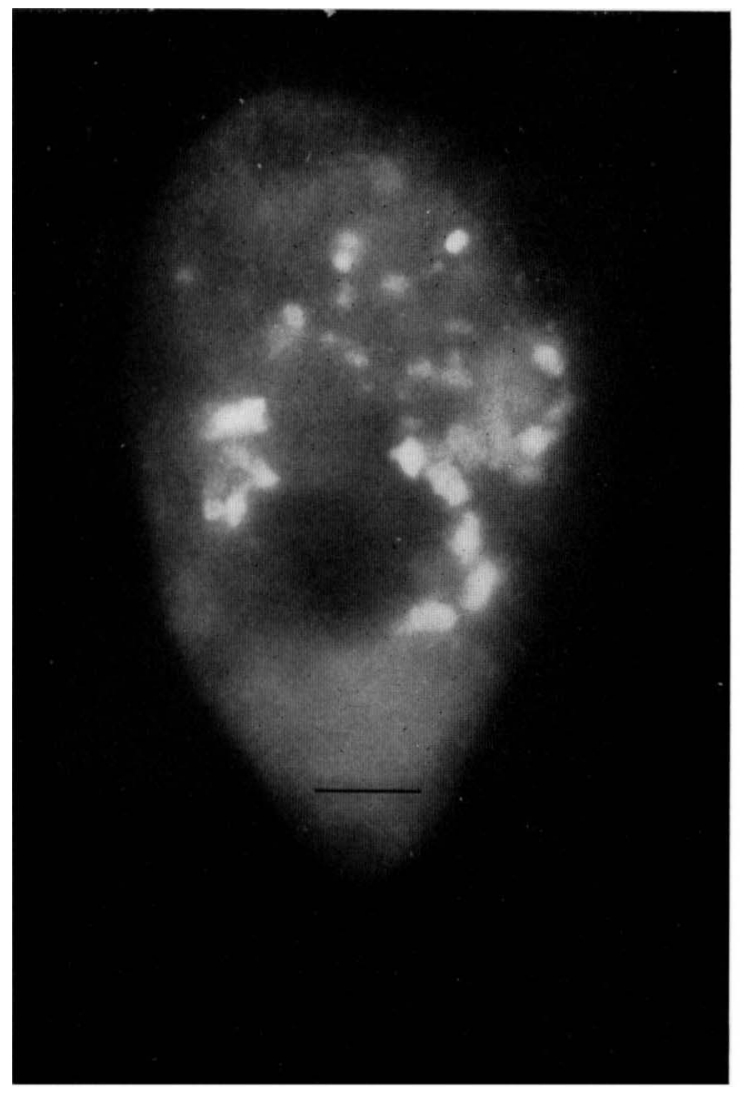

Fig. 1

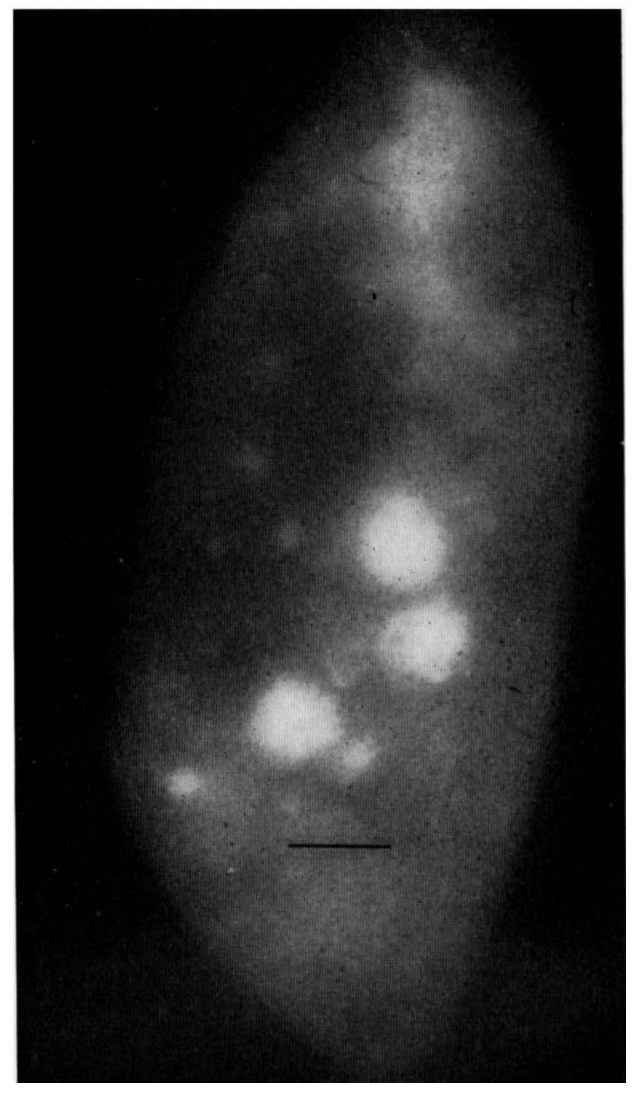

Fig. 2 